\title{
Channel Estimation of DWT Based MIMO-OFDM System in Wireless Communication
}

\author{
Nivedita Singh and Manoj Kumar Shukla* \\ Electronics Engineering Department, HBTU, Kanpur, Uttar Pradesh, India; \\ nivedita.sakshi@gmail.com,mkshukla@hbtu.ac.in
}

\begin{abstract}
Objectives: The study is to analyse the $5 \times 5$ wavelet based Multiple Inputs Multiple Outputs (MIMO)-Orthogonal Frequency Division Multiplexing (OFDM) system, considered to observe the system BER performance and system capacity for higher count of transmitter/receiver compared to conventional systems (i.e. $2 \times 2$ ). Methods analysis: This study basically involves the Least Square channel estimation method which helps to easily equate the higher number of transmitter and receiver. With the FFT Tool being used in OFDM systems, the problem of the higher bit error rate was still the matter of concern. To further avoid this problem, researcher's has adopted a better method called as Wavelet Transform Tool. The results have been discussed by using BER versus SNR graph. The simulation results are being performed on MATLAB platform of R2016a version. Findings: The simulation results, have the wavelet based MIMO-OFDM system with and without channel coding, which shows that a system with channel coding has better BER performance than system without channel coding because it helps to detect and correct the errors. The simulation results for throughput performance of the system with different modulation order/schemes, and code rate shows that there is a trade-off between the BER performance and code rate of the system. When the code rate and modulation order of a system increases, there is an increase in capacity to carry more information at a time along with the tendency of occurring error too. The proposed work is being compared with the conventional system $(2 \times 2)$ showing use of higher count of transmitter/receiver helps in transmitting more bits at a time but with a drawback of higher BER. It can further be the area of research of transmitting data by multiple users simultaneously, using most appropriate tool/methods that cause better system performance. Application: This study results can be used for further analysis in the wireless communication field and for achieving higher data rate for multiple users.
\end{abstract}

Keywords: Multiple Inputs Multiple Outputs, Orthogonal Frequency Division Multiplexing, Intersymbol Interference, Discrete Wavelet Transform.

\section{Introduction}

Wireless communication is a term that allows all the procedures and form of connecting and communicating between the several networks wirelessly. The main objective of this technology is to provide larger area coverage, high quality data rate services, and better spectral efficiency, but the wireless network is adversely affected by the multipath interference. To attain the high performance and robustness in our communication system, the channel estimation scheme is required. 1 The impulse response of the channel will help in knowing the important information for designing and planning of wireless systems. ${ }^{2}$ This article considers Least Square channel estimation method which is a training sequence based channel estimation technique.

In order to eliminate the Intersymbol Interference (ISI) effect, Orthogonal Frequency Division Multiplexing (OFDM) is being used. It is a combination of multiplexing and modulation both. It divides the available spectrum into multiple sub-channels and converts the frequency selective channel to multiple flat fading channels for higher data transmission rate. Because of this, the symbol duration get larger than the delay spread of the channel,

${ }^{*}$ Author for correspondence 
which helps to handle ISI. OFDM digital implementation is possible by using a mathematical tool called as FFT and IFFT. Cyclic prefix is like a guard interval which is inserted in the OFDM symbol in order to avoid further the ISI problem, but has drawback of decrease in the spectral efficiency and high power consumption. $\underline{3}$ To create multidimensional wireless communication system, Multiple Inputs Multiple Outputs (MIMO) has been considered, a multiple antenna system with an efficient spatial diversity technique which enhances the multipath propagation in order to increase range, capacity, and reliability of wireless communication system. $\underline{4}$

In the proposed work, $5 \times 5$ DWT based MIMOOFDM system in AWGN channel has been considered for observing the performance of system with higher counts of transmitter and receiver against the noise and interference. In the literature, the $2 \times 2$ system was considered to be the conventional by the researchers. In this paper, the least square channel estimation has been adopted to estimate the channel parameters as it is supposed to be better in computing channel coefficients for higher number of transmitter/receiver. In this paper, MIMO-OFDM system is considered in which the FFT and IFFT tool in OFDM system get replaced with DWT and IDWT, respectively, in order to attain much higher spectral efficiency than the conventional one. The introduction section of this paper includes the literature survey also, which demonstrates existing FFT-OFDM system. Further in section 2, the proposed model DWT-OFDM system with MIMO is shown. The DWT-OFDM system is more robust against multipath fading effects andinterference as compared to

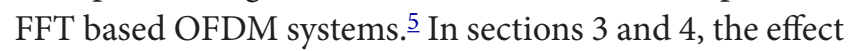
of CP on spectral efficiency of system and the LS channel estimation technique has been discussed, respectively. At last in sections 5 and 6 , the results based on MATLAB simulation have shown and the conclusion is drawn based on the computed results.

The MIMO-OFDM system proves to be more efficient than the other proposed systems. The MIMO block focuses on sending signals of multiple users at a time by using same channel bandwidth. The problem of ISI is resolved with the help of OFDM part and available spectrum is being utilized efficiently by multiple users with the help of MIMO system. $\cdot$ During transmission, the multipath propagation is perceived as interference, degrading a receiver's ability to recover the transmitted signal, however, the MIMO system utilizes this disability to increase throughput and reliability of the system. ${ }^{6}$ It allows multiple users in a single channel to work parallely. The MIMO system spreads the same total power over different antennas to achieve higher array gain which helps to improve the spectral efficiency.

To achieve the required bit rate, OFDM multicarrier transmission systems has been adopted, as it follows the basic principle of orthogonality to avoid ISI and ICI, in which the subcarriers are spaced closely in frequency domain without interfering to each other. Previously, multiple sinusoidal generators were used to generate these orthogonal sub-carriers, because of which the OFDM system has large and complex structure. To avoid this complexity, researchers started using DFT instead of sinusoidal generators for getting the sub-carrier. Furthermore, researcher adopted new mathematical tool FFT instead of DFT, as FFT-based OFDM structure has less computational complexity. The basic idea behind this is to transmit the each single information stream onto the separate carrier parallelly. It allows transmission of multiple information streams at a time without interference of each other in a lesser bandwidth.

To generate the OFDM signal, first consider the spectrum required by the input data and the modulation scheme which is used for symbol mapping. The bit stream of input signal is encoded, where the redundant bits are added for security and reliability reason. These encoded bits are passed through the serial to parallel buffer stage so that it divides the single stream information into multiple sub-streams. Furthermore, this parallel binary generated data is mapped and converted into a waveform pattern with the help of modulation scheme (BPSK, QPSK, and QAM). This is done to easily load that data onto the analog carrier and to easily identify the error pattern with the help of constellation diagram. The phase and amplitude of carrier is being decided with the help of modulation scheme. Each sub-stream is being modulated onto a subcarrier by IFFT operation.

The problem of ISI occurs when the transmitted symbol interferes with itself and receivers do not decode the transmitted signal correctly. To resolve this issue, symbols are padded with guard interval named as cyclic prefix, which acts a buffer region to save the transmitted OFDM symbol from ISI. $\underline{3}$ This cyclic prefix transforms the linearly convolutional channel to cyclic convolutional channel. The OFDM transmitter and receiver can be seen in Figure 1. The signal is passed through the AWGN/ Rayleigh channel and received at the receiver end. In order to reconstruct signal back, there is removal of cyclic 


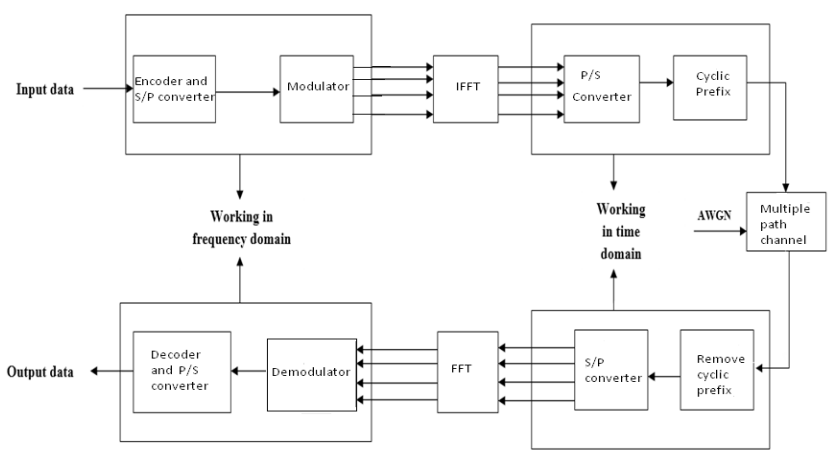

Figure 1. FFT-based OFDM transceiver system.

extension, which converts the received symbol parallelly again by serial to parallel buffer and demodulate it by using the FFT tool. Equation for IFFT is

$$
\mathrm{x}(\mathrm{n})=\frac{1}{N} \sum_{l=0}^{N-1} X(l) e^{i^{*} 2^{*} p^{*} l^{*} n / N}
$$

Equation of FFT is

$$
\mathrm{x}(1)=\sum_{k=0}^{N-1} x(n) e^{-i^{*} 2^{*} p i^{*} l^{*} n / N}
$$

Here, $N$ denotes the total number of sub-carrier, and 1 denotes the lth sub-carrier on which the data are being loaded.

\section{DWT Based MIMO-OFDM System}

In the proposed system, the basic principle of DWTMIMO-OFDM system has taken into consideration, in which the IDWT tool is used for modulation and DWT tool is used for demodulation. It is an additional approach to study signal in both time and frequency domain. ${ }^{6}$ The wavelet transform is a more preferred tool over Fourier transform as it has better frequency and time localisation. It has been considered better for the study of non-stationary signals. They both satisfy the rule of orthogonality and used as a carrier in OFDM system. Wavelet basically means a small (finite length) oscillatory wave with amplitude that starts at zero, increases, and again settles down to zero. The wavelet transform is a mathematical tool used to give time-frequency representation of signal. ${ }^{7}$ It helps to analyse at what interval of time, the signal has that frequency component. Generally, it has categorized into two formats one is the continuous wavelet transform (CWT) and the other one is discrete wavelet transform (DWT). DWT does not mean evaluation of signal is being done in discrete domain, whereas it symbolizes the evaluation of signal is being taken in continuous domain with the discretisation of scaling and translational parameters. The CWT tool is being avoided in OFDM as it evaluates the coefficients on each scale causing numerous unwanted coefficients to represent the signal, which increases the redundancy and complexity at receiver side.

The wavelet basis function is given by $\underline{?}$

$$
W(s, \tau)=(1 / \sqrt{s}) \psi((t-\tau) / s)
$$

It dilates, translates and scaled the mother wavelet $\psi(t) .{ }^{7}$ Here, $s$ is a scaling parameter decides the width of the wavelet, and $\tau$ is the translating parameter decides the duration of the wavelet. The above equation is representation of CWT basis function. For DWT, the researchers have derived the basis function with the help of CWT, by putting some specified value, which evaluates the coefficient for representing the signal at some specific scale. It follows the Heisenberg uncertainty principle which states that one cannot know what spectral component can exists in what instances of time; we can only have the information about the time interval in which certain band of frequency exists.

DWT mainly decompose the signal into a set of mutually orthogonal wavelet basis functions and has filters of different cutoff frequencies which is used to analyse the signals at different scales. $\frac{8}{-}$ The signal is decomposed into successive bands from which one is passed through a low pass filter and the other one is passed through a high pass filter repeatedly. $\frac{3}{\text { Th }}$ The low passed one produces approximation that is eliminating the high frequency component, leaving out the details and considering only approximate part of signal so they are called as approximate coefficients. The signal which is passed through a high pass filter is used to analyse the high frequencies generating the detail coefficient leaving out the approximate part of signal by considering the detailed one only. Each level gives the detailed and approximated coefficients, till the further decomposition of signal is not possible. Figure 2 represents the basic DWT/IDWT structure, and Figure 3 shows the three level decomposition of signal having a series of low pass and high pass filter.

Equation for IDWT of $\mathrm{X}(\mathrm{K})$ is given by ${ }^{9}$

$$
x(n)=\sum_{m=-\infty}^{\infty} \sum_{k=-\infty}^{\infty} X(m, k) 2^{m / 2} \psi\left(2^{m} n-k\right)
$$




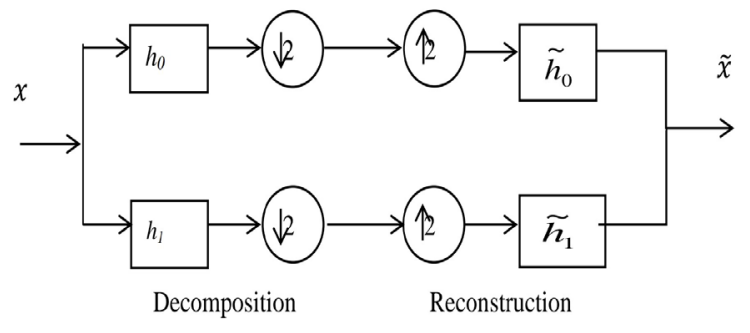

Figure 2. Decomposition and reconstruction of signal using DWT and IDWT.

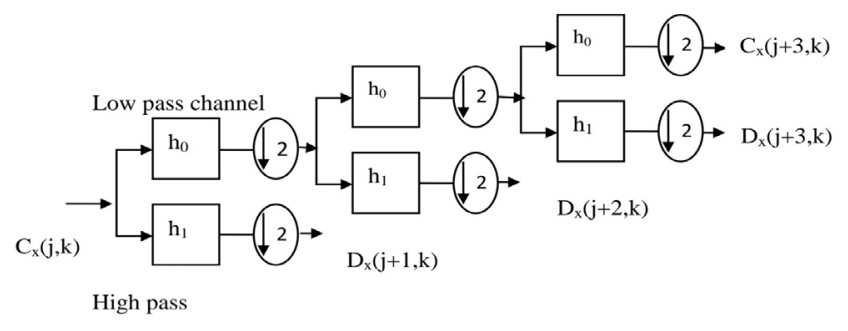

Figure 3. DWT structure.

Equation for DWT of $\mathrm{x}(\mathrm{n})$ is given by $\underline{10}$

$$
X(m, k)=\sum_{n} x(n) 2^{m / 2} \psi\left(2^{m} n-k\right)
$$

The scale is changed by up-sampling and downsampling (sub-sampling) operations. It reduces some sample of the signal. $\psi(\mathrm{m}, \mathrm{k})$ is the mother wavelet function, $m$ represents the translation scale. Figure 4 shows the transmitter/receiver structure for DWTMIMO-OFDM system. The input bits are first taken by the encoder to encode the bit stream with a particular code rate. Then the encoded stream is sent to the modulator for converting the input binary stream to waveform pattern.

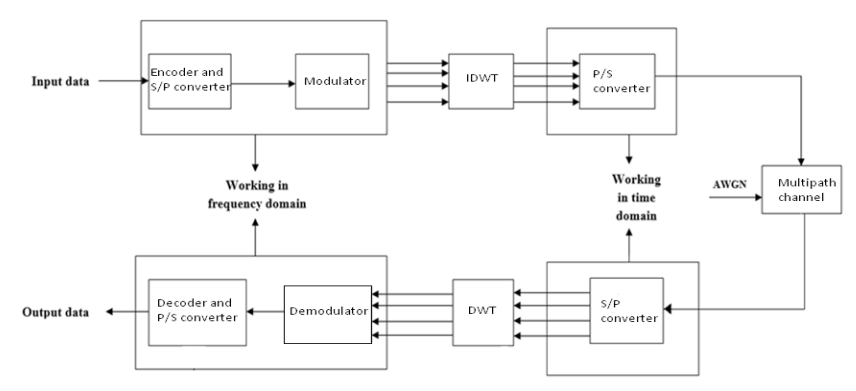

Figure.4. DWT-based OFDM transceiver system.
Again the symbol is passed through the serial to parallel buffer for further transmission. The symbol gets loaded to M-band wavelet carrier with the help of IDWT tool. These symbols are transmitted through the channel and get demodulated by using the DWT tool at the receiver end.

\section{Cyclic Prefix}

Due to signal propagation phenomena, such as reflection, diffraction, or say multipath, a receiver can receive several delayed versions of the same signal. At the receiver, all these multipath components are summed and multiple symbols being received simultaneously overlap with each other called as ISI. If the bandwidth of the transmitted signal is very large compared to the bandwidth of channel, then there will be effect of interference in the symbol. But if the symbol length gets increase (or can say bandwidth of transmitted symbol get decreases), then the effect of ISI can be mitigated. In order to increase the symbol length, the copy of last part of symbol is being inserted at initial point of symbol as shown in Figure 5. The receiver can identify the end points of each symbol and correctly correlates the information and helps to eliminate the ISI.

$N_{\text {cp }}$ denotes the length of the cyclic prefix, $N_{\text {fft }}$ is the IFFT window length, and $N$ show the number of sub carriers. It compensates for ICI and ISI but it consumes a considerable amount of spectrum and power. 11 Assuming the channel having maximum delay of $\mathrm{m}$, insert cyclic prefix of duration $n$ greater than channel impulse response $\mathrm{m}$. It consumes large transmissionenergy and cause a loss in data rate of

$$
\frac{\text { Cyclic prefix }(\mathrm{n}) \times 100 \%}{\text { Cyclic prefix }(\mathrm{n})+\text { Number of subcarrier }(\mathrm{N})}
$$

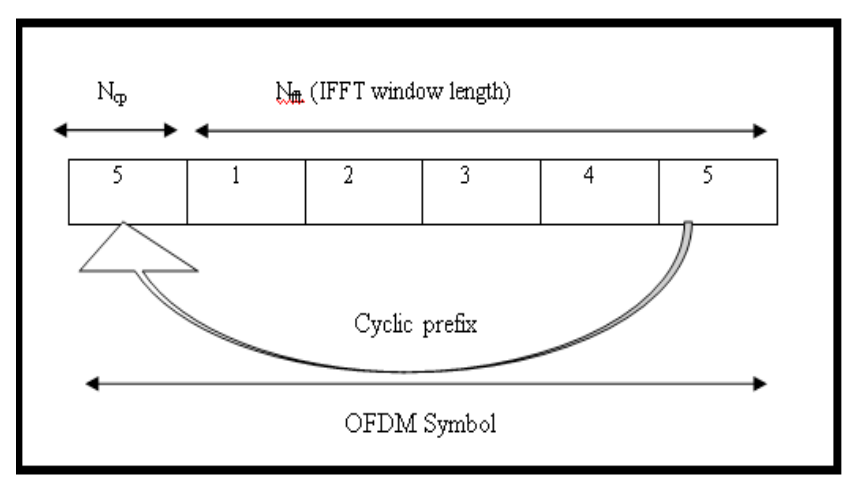

Figure 5. OFDM symbol for FFT. 
It reduces spectral efficiency and data rates because it does not contain any information. As, increase in length of symbol further to overcome ISI, it reduces the overall power efficiency and disperses the transmitter energy results in loss of SNR which is given by

$$
-10 \log _{10}\left(\frac{\text { TotalOFDM symbollength-Cyclic Prfix Length }}{\text { TotalOFDM symbollength }}\right)
$$

The waveform being used in wavelet transform are longer than the normal transform duration, because of which DWT-OFDM symbols overlap in time domain and gives better frequency localisation. In result, it does not require a cyclic prefix to extend the symbol duration in order to mitigate ISI. In addition, FFT-OFDM symbols are passed through rectangular shape of the window generating larger side lobes, this is avoids in case of DWT-OFDM. Moreover, it reduces the complexity of system from FFTs $O\left(\mathrm{Nlog}_{2} \mathrm{~N}\right)$ to $O(\mathrm{~N}) . \underline{12}$ The DWTOFDM not only saves the bandwidth by not adding CP to OFDM symbol but also saves the transmission power and enhances the capacity of system.

\section{Channel Estimation}

Channel estimation is a mathematical estimation of what is exactly happening in the environment. It allows receiver to approximate and measure the effect of channel on signal, so that ISI and noise can be removed from it. $\underline{13}$ In proposed model pilot sequence based channel estimation called as Least Square Channel estimation technique has been considered. In this, a reference carrier named as a pilot symbol is known at the receiver end in terms of position or pattern and used for channel estimation. The transmitted and received signal is represented in vector and matrix form for the ease of evaluation. For any lth subcarrier, the received vector can be represented as

$$
Y(l)=X(l) H(l)+W(l)
$$

$\mathrm{H}(\mathrm{l})$ represents the channel coefficient across lth subcarrier and $\mathrm{X}(\mathrm{l})$ represents the symbol loaded onto lth subcarrier. For $\mathrm{N}$ subcarrier after rearrangement, the received signal in matrix form can be represented as

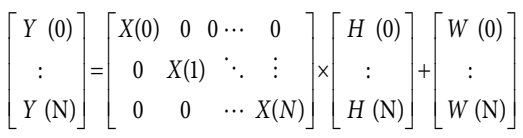

$\mathrm{Y}$ is $\mathrm{N} \times 1$ receiver pilot output matrix in subcarrier domain, $\mathrm{X}$ is $\mathrm{N} \times \mathrm{N}$ diagonal invertible matrix whereas $\mathrm{W}$ is a $\mathrm{N} \times 1$ noise matrix in sub carrier domain. Estimate of coefficient on lth subcarrier is given by

$$
\hat{\mathrm{H}}=X^{-1} Y
$$

where $\mathrm{X}^{-1}$ is the pseudo-inverse of $\mathrm{X}$ and is given by

$$
\left[\begin{array}{ccccc}
\frac{1}{X(0)} & 0 & 0 & \cdots & 0 \\
0 & 0 & \vdots & \ddots & 0 \\
0 & 0 & & \cdots & \frac{1}{X(N)}
\end{array}\right]
$$

So, by above discussion FFT of channel coefficients has been found. In order to find the channel taps estimate take IFFT of the estimate of subcarrier coefficient.

$$
\hat{\mathrm{H}}=Y(l) / X(l)
$$

\section{Simulation Results}

DWT based MIMO-OFDM system performance has been measured in the SNR verses BER graph. The required simulation parameters are shown in Table 1 and their performance is being compared in figures shows inverse relation in SNR and BER, an increase of SNR the noise content in signal decreases and the probability of occurring error also decreases. In Figure 6, the five transmitter and five receiver antennas perform this communication process without channel coding resulting in increase in BER as increase of order of modulation scheme. QPSK has lowest BER compared with 16-QAM and 64-QAM, which also matches to the theoretical reason of sending lesser bits at a time results in lower tendency of occurring error.

Figure 7 shows performance and comparison of system with convolutional coding with two different rates and modulation scheme. It is said to be convolutional

Table 1. System specification table

\begin{tabular}{|l|c|}
\hline Parameter & $\begin{array}{c}\text { Specification DWT-MIMO- } \\
\text { OFDM system }\end{array}$ \\
\hline Channel model & AWGN channel \\
\hline Modulation & QPSK, 16-QAM, 64-QAM \\
\hline Diversity scheme & $5 \times 5$ \\
\hline Channel estimation & Least square \\
\hline Number of subcarriers & 1024 \\
\hline Code rate & $1 / 2$ and $3 / 4$ \\
\hline
\end{tabular}




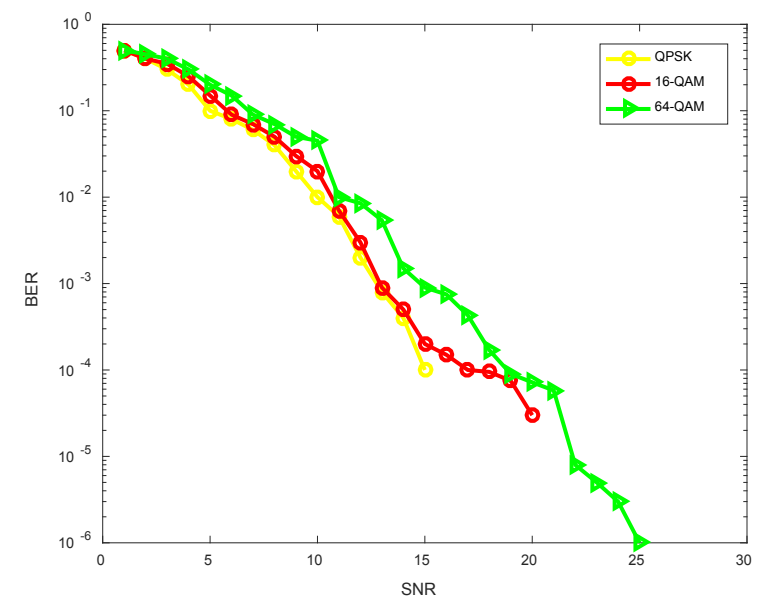

Figure 6. BER performance of system without channel coding.

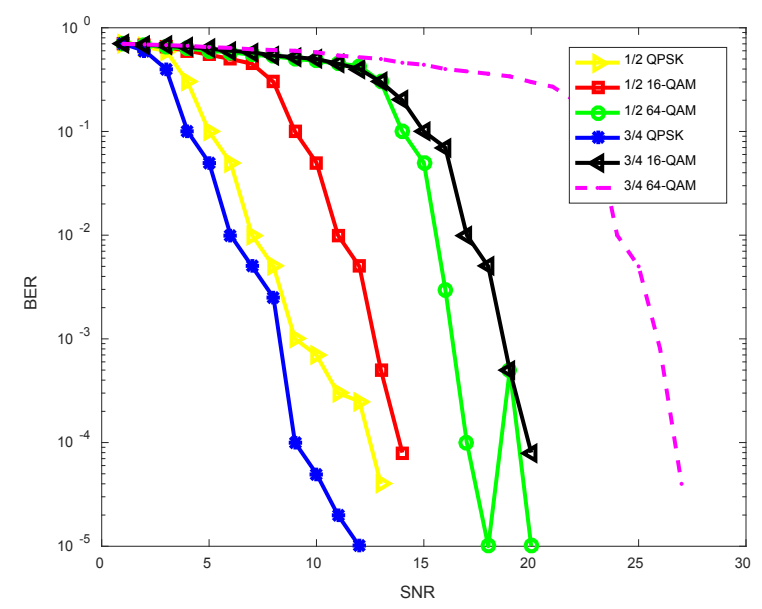

Figure 7. BER performance of system with convolutional channel coding.

encoder as it performs a convolutional of input streams with encoder impulse response. As, channel codes adds redundancy to the codes provide protection to the information bits which enhanced the BER performance of signal. The BER performance of QPSK, 16-QAM, 64-QAM with code rate of $1 / 2$ and $3 / 4$ follows the same order for occurrence of error as shown in Figure 8. As, for lower code rate means higher the number of bits for data error detection and correction process, so performance of the system enhances but have less bandwidth efficiency. For higher code rate and order of modulation of system greater will be the rate of occurrence of error, so 64-QAM with code rate $3 / 4$ has the higher probability of error.

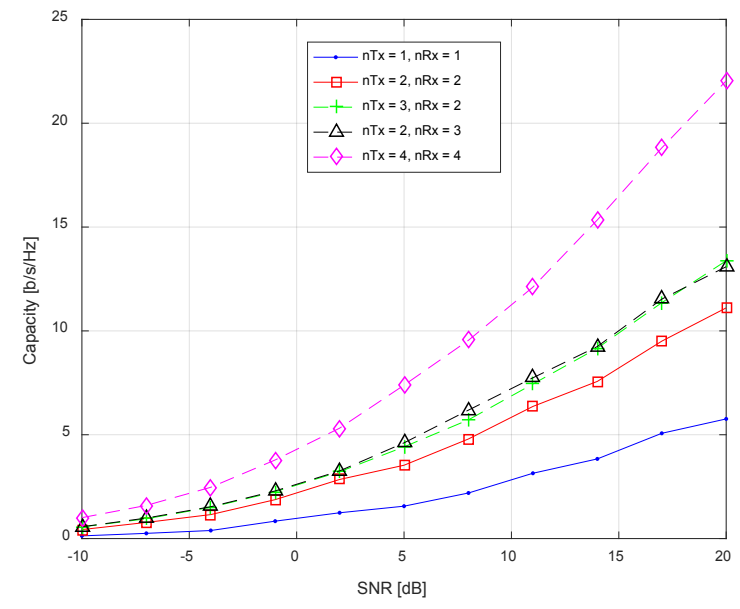

Figure 8. Average capacity of a MIMO system for different number of Tx-Rx antennas.

Table 2. DWT-MIMO-OFDM system comparision table

\begin{tabular}{|l|c|c|}
\hline & $\begin{array}{c}\text { Specification } \\
\text { DWT-MIMO- } \\
\text { OFDM system }\end{array}$ & $\begin{array}{c}\text { Specification(proposed } \\
\text { model) DWT-MIMO- } \\
\text { OFDM system }\end{array}$ \\
\hline $\begin{array}{l}\text { Channel } \\
\text { model }\end{array}$ & AWGN channel & AWGN channel \\
\hline Modulation & QPSK & QPSK, 16-QAM, 64-QAM \\
\hline $\begin{array}{l}\text { Diversity } \\
\text { scheme }\end{array}$ & $2 \times 2$ & $5 \times 5$ \\
\hline $\begin{array}{l}\text { Channel } \\
\text { estimation }\end{array}$ & Least square & Least square \\
\hline Code rate & $1 / 2$ & $1 / 2,3 / 4$ \\
\hline
\end{tabular}

Table 2 refers for the comparison among considered conventional system $(2 \times 2)$ and the proposed system $(5 \times 5)$. The results show that as the number of transmitter and receiver increases, then the performance of the system gets degraded. For proposed system, BER has increased compared to the conventional one. Major effect on system can be seen for lower SNR values, as the noise content in signal is higher, chances of occurring error is high for any system. As, on increase in transmitting antennas, it results in transmission of more bits for multiple user (by using MIMO scheme) independently causes more possibility of occurring errors, which can be seen in simulation results.

Throughput is an important indicator of performance and quality of network connection. It is the rate of 


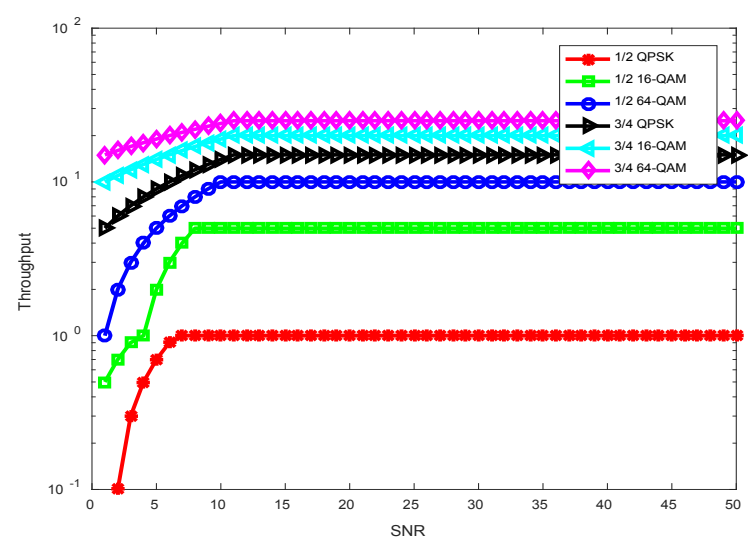

Figure 9. Throughput performance of system with channel coding.

successful message transmission over a communication channel. Figure 9 shows the throughput performance of system with different code rate and modulation schemes. It indicates that higher the code rate and order of modulation scheme better will be the throughput performance of system. Theoretically, it is being measured with the Shannon formula. At the lesser value of SNR system did not perform well, but as SNR got improved the rate of successful transmission of data enhances resulting in good throughput performance of system. After a certain range of SNR, the performance of the system gets constant.

Figure 8 represents the average capacity of a MIMO system for different number of transmitter/receiver antenna. Furthermore, the capacity versus SNR graph has been shown for the analysis. As, the number of transmitter/receiver increases, capacity to carry more information (bits) also increases. Hence, there is proper channel utilisation. This paper includes the water filling algorithm for allocating the optimal transmitting power for independent multiple parallel channels, computing the capacity of the MIMO channel for a given noise power of $10^{-4}$ watt and SNR range of $-10 \mathrm{~dB}$ to $20 \mathrm{~dB}$. As, the SNR range of $-10 \mathrm{~dB}$ to $50 \mathrm{~dB}$ is considered suitable for voice and wireless network, that is why this paper includes the above stated SNR range. The $3 \times 2$ and $2 \times 3$ diversity scheme has almost same capacity for carrying information however, $4 \times 4$ has the maximum one. The total capacity for a MIMO channel is given by Shannon channel capacity theorem

$$
\sum_{i=1}^{t} \log _{2}\left(1+P_{i} \sigma_{i}^{2} / \sigma_{n}^{2}\right) b i t s / s / H z
$$

Here, $t$ is the number of transmitting antenna $\sigma_{i}^{2}$ is gain of $i$ th stream of channel obtained by th $\sigma_{n}^{2}$ e singular value decomposition of channel matrix, is power of noise present in the channel and $P_{i}$ denotes the transmitted power of the $i$ th signal. Here, $P_{\mathrm{i}}$ is calculated by water filling algorithm, where

$$
P_{1}+P_{2}+P_{3} \ldots \ldots \ldots \ldots P_{t}<P_{\text {total }}
$$

and $P_{\mathrm{i}}$ is $\left(1 / \mathrm{X}-\sigma_{n}{ }^{2} / \sigma_{i}^{2}\right)$, here $\mathrm{X}$ is Lagrange's multiplier.

\section{Conclusion}

The transmission rate of DWT-MIMO-OFDM system gets improved by the dynamic adjustment of radio transmitter modulation schemes, which ensures the higher data throughput and spectral efficiency with higher order of modulation. The primarily defined DWT tool in place of FFT tool helps to avoid insertion of cyclic prefix, that leads to bandwidth and power saving of a wireless communication system. The simulation results of the proposed system with and without channel coding has been demonstrated showing channel coding helps to detect and correct the errors, causing decrease in BER performance of the system. The simulation results for throughput performance of the system with different modulation order/schemes, and code rate shows that there is a trade-off between the BER performance and code rate of system. As, on increase in code rate and the count of transmitter and receiver there is increase in carrying more number of bits through available channel and also in probability of occurring error. So, by using higher count of transmitter/receiver helps in transmitting more information at a time but with a drawback of higher BER.

\section{References}

1. Sonali AB, Patil BP, Bhalchandra SS. Performance of MMSE channel equalization for MIMO-OFDM system. In: International conference of computing communication control and automation; 2016.

2. Liang P, Yuan F, Wei L, Zhisen W. Channel estimation in mobile wireless communication. In: International conference on communication and mobile computing; 2010. P. $77-80$. 
3. Meenakshi D, Prabha S, Raajan NR. Compare the performance analysis for FFT based MIMO-OFDM with DWT based MIMO-OFDM. In: IEEE international conference on emerging trends in computing, communication and nanotechnology; 2013. P. 441-5.

4. Chaudhary RS, Patil Anil J, Ashwini VY. WLAN-IEEE 802.11 ac: Simulation and performance evaluation with MIMO-OFDM. In: Conference on advances in signal processing; 2016. P. 440-45.

5. Akhilesh V, Krithika V, Partibane B. Channel estimation for a multi-user MIMO-OFDM-IDMA system. In: International conference on communication and signal processing; 2017. P. 1823-6.

6. Vishal S, Harleen K, Gurpreet S. BER assessment of MIMO incorporated W-OFDM wireless communication system. In: International conference on advanced computing and communication technologies; 2015. P. 463-6.

7. Oboyerulu EA, Idowu-Bismark Olabode B, Ibhaze Augustus E. Comparative analysis of fast Fourier transform and discrete wavelet based MIMO-OFDM. Int J Commun Antenn propag. 2017;7(2):168.
8. Abhishek G, Prateek N, Vijayshri C. A review on wavelet transform as a substitute to cyclic prefix removal in FFT in OFDM. Int J Eng Sci Res Technol. 2014;3(2):827-30.

9. Marius O, Miranda N. Errors per scale statistics for a wavelet OFDM transmission in flat fading channels. In: $6^{\text {th }}$ IEEE international symposium on intelligent signal processing; 2009. P. 119-24.

10. Broughton SA, Bryan K. Discrete Fourier analysis and wavelets. New Jersey: John Wiley. 2009. P. 354.

11. Amar AJ, Iviva K. Cyclic prefix length determination for orthogonal frequency division multiplexing system over different wireless channel models based on maximum excess delay spread. Am J Eng Appl Sci. 2015;8(1):82-93.

12. Kumar GM, Sarika S, Raghuvanshi AS, Tiwari S. Channel estimation for wavelet based OFDM system. In: International conference on devices and communications; 2011.

13. Qiao YT, Yu S, Su P, Zhang L. Research on an iterative algorithm of LS channel estimation in MIMO OFDM systems. IEEE Trans Broadcast. 2005;51(1):149-53. 